\title{
An Exploration of Patient Navigation and Community Health Worker Activities Across National Comprehensive Cancer Control Programs
}

\author{
Elizabeth A. Rohan,,* Renee McDougall, and Julie S. Townsend ${ }^{1}$
}

\begin{abstract}
Purpose: Health disparities persist across the cancer care continuum. Patient navigator (PN) and community health worker (CHW) interventions are designed to increase health equity. National Comprehensive Cancer Control Program (NCCCP) awardees develop and implement plans to coordinate cancer prevention and control activities, including supporting PN and CHW interventions. This content analysis examined NCCCP action plans to assess the extent to which jurisdictions report engaging in PN and/or CHW activities.

Methods: We abstracted PN and CHW content from NCCCP action plans and coded content according to specific areas of PN and/or CHW intervention (e.g., screening, survivorship, and cancer type), used descriptive statistics to characterize overall results, and calculated chi-squares to determine whether programs engaged PNs and CHWs differently.

Results: Eighty-two percent $(n=53)$ of 65 NCCCP action plans had content related to PN and/or CHW activities, with more PN language (83\%) than CHW (58\%). These action plans described engaging PNs and CHWs in activities across the cancer continuum, but particularly for screening (60\%) and survivorship (55\%). Eighty-one percent of these plans described activities related to workforce development, such as training and standardizing roles and competencies. Programs engaged CHWs more often than PNs for outreach and in community settings.

Conclusion: The majority of NCCCP awardees reported engaging in PN and/or CHW activities. Understanding how NCCCP awardees engage PNs and CHWs, including awardees' needs for workforce development in this area, can help Centers for Disease Control and Prevention provide more focused technical assistance as programs increase engagement of PNs and CHWs to improve health equity.
\end{abstract}

Keywords: community health workers; comprehensive cancer control; disparities; patient navigation

\section{Introduction}

Racial and ethnic minorities, rural communities, and those with low socioeconomic status, including those who are un- or under-insured, bear a disproportionately high burden of cancer in the United States. ${ }^{1-4}$ These disparities persist across the cancer continuum, from prevention to screening, treatment, and longterm survivorship. ${ }^{4-6}$ Barriers to cancer prevention, screening, and care often include fear or distrust of the medical community, lack of or inadequate health insurance coverage, financial hardship, low levels of health literacy and education, and logistical barriers, such as lack of transportation. ${ }^{7,8}$ The social determinants of health, that is, the social, economic, cultural, and physical contexts within which people live, are considered important when addressing health disparities. ${ }^{9-11}$

Patient navigation (PN) and community health worker $(\mathrm{CHW})$ interventions have been demonstrated

\footnotetext{
'Division of Cancer Prevention and Control, Centers for Disease Control and Prevention, Atlanta, Georgia.

${ }^{2}$ Surgery Department, Animal Medical Center, Manhattan, New York.

*Address correspondence to: Elizabeth A. Rohan, PhD, MSW, Division of Cancer Prevention and Control, Centers for Disease Control and Prevention, 4770 Buford Hwy, NE, MS F-76, Atlanta, GA 30341, E-mail: erohan@cdc.gov
}

(C) Elizabeth A. Rohan et al. 2018; Published by Mary Ann Liebert, Inc. This Open Access article is distributed under the terms of the Creative Commons License (http://creativecommons.org/licenses/by/4.0), which permits unrestricted use, distribution, and reproduction in any medium, provided the original work is properly cited. 
to reduce health disparities by addressing the social determinants of health. ${ }^{9,12-18}$ Cancer $\mathrm{PN}$ is a process that provides "individualized assistance to cancer patients, families, and caregivers to help overcome healthcare system barriers and facilitate timely access to quality health and psychosocial care from pre-diagnosis through all phases of the cancer experience." ${ }^{\prime 9}$ PNs assist patients in overcoming barriers to cancer screening and treatment, offer peer counseling, provide linkages to financial and community resources, and provide culturally competent patient education, particularly among (but not limited to) the underserved. ${ }^{20}$ Studies have shown the effectiveness of $\mathrm{PN}$ in increasing patient completion of cancer screening and reducing disparities among the medically underserved. ${ }^{12,13,21,22} \mathrm{~A}$ recent systematic review of both $\mathrm{PN}$ and $\mathrm{CHW}$ interventions at Federally Qualified Health Centers, which provide primary care to medically underserved communities, found that $\mathrm{PN} / \mathrm{CHW}$ interventions were effective in increasing cancer screening rates. ${ }^{9}$ Similarly, a systematic review of CHW interventions and mammography found that such interventions improve mammography rates. ${ }^{17}$

Dr. Harold Freeman pioneered PN in 1990 to address the challenges low-income, medically underserved populations experience with access to timely cancer screenings, treatment, and care. ${ }^{13} \mathrm{PNs}$ often come from the community they serve, but they typically function in clinical settings to reduce barriers to cancer screening and timely treatment, act as liaison to the medical team, and help cancer patients and families manage the logistics of cancer treatment, which often includes various specialists and time-intensive treatment. Depending on the needs of the health care setting, PNs may be lay members of the community they serve or health care professionals (usually nurses or social workers). ${ }^{23}$ Their scopes of practice are reflective of their backgrounds. ${ }^{24}$

CHW programs gained popularity in the United States in the 1960s, but their international origins date back to the 19th century. ${ }^{15} \mathrm{CHWs}$ are frontline public health workers who are trusted members of the community they serve and provide the cultural expertise to help link the community to health and social services. ${ }^{9,15,25-27}$ Depending on community or context, CHWs are also known as community health advisors, outreach workers, community health representatives (CHRs), promotoras(es), peer counselors, lay health advocates, and peer health educators, among other titles. $^{28,29}$ Historically, CHWs have worked through community-based organizations focusing on health education, prevention, and screening, while PNs have functioned in clinical settings to navigate cancer patients through the health care system, ${ }^{23}$ although recent trends using $\mathrm{CHWs}$ within health care teams and navigators for outreach have blurred those boundaries. ${ }^{30-32}$

PNs and CHWs have become increasingly valued members of the U.S. health care workforce in addressing health disparities and other public health priorities, particularly following changes in health care payment structures and grant funding under the Patient Protection and Affordable Care Act, ${ }^{33,34}$ in addition to recommendations and guidance contained in the U.S. Department of Health and Human Services' National Prevention Strategy. ${ }^{35}$ Since 2015, the American College of Surgeon's Commission on Cancer has required PN services as a component of accreditation. ${ }^{13}$

The Centers for Disease Control and Prevention (CDC) funds the National Comprehensive Cancer Control Program (NCCCP) in all 50 states, the District of Columbia, and several tribes, tribal organizations, U.S. territories, and Pacific Island Jurisdictions to broadly promote cancer prevention and control activities and interventions. ${ }^{36}$ NCCCP awardees focus their activities on the following priority areas: emphasizing primary prevention of cancer; assisting with coordination of early detection and treatment activities; addressing public health needs of cancer survivors; helping with using policy, systems, and environmental changes (PSEs) to guide sustainable cancer control; and promoting health equity and reducing disparities. ${ }^{37}$ Each NCCCP awardee works with a coalition and other stakeholders to develop and implement comprehensive cancer plans, which are blueprints for addressing their population's particular cancer burden, and they document progress in yearly action plans. ${ }^{38}$ Since PNs and CHWs could be used to address most of the NCCCP priority areas, we sought to examine the extent to which awardees are engaging in activities related to and supportive of $\mathrm{PN}$ and/or CHW interventions. The objective of this content analysis was to examine NCCCP action plans for incorporation of PN and/or CHW interventions in cancer control.

\section{Methods}

We abstracted content from NCCCP action plans $(n=65)$ in December 2015 from CDC's Chronic Disease Management Information System (CDMIS; www .cdc.gov/cdmis/index.html) using these keywords: navigate, navigator(s), navigation, peer educator, community 
navigator, community health aid, lay health worker, Promotora(s), peer counselor, peer leader, lay health educator, CHWs, PNs, CHRs, lay health advisor, peer health advisor, peer health educator, community health advisor, and outreach worker. The NCCCP action plans spanned a 3-year time period, beginning in 2012 at the onset of a new 5-year funding cycle (2012-2017). As part of funding expectations, NCCCP awardees submit via CDMIS annual action plans that outline specific objectives and activities to be accomplished in the upcoming year, and report on progress they made during the current project year for each action plan objective. CDMIS meets all federal requirements and approvals for public data collection (OMB No. 0920-0841).

After abstraction, we deleted duplicate text entries and reviewed action plan text for inclusion, based on where in the action plan the term appeared. Only plans that used the keyword in text as part of an objective, strategy, or goal were included. For example, we excluded content describing webinars attended by NCCCP awardees on PN/CHW topics or descriptions of coalition members who were PNs or CHWs, without reference to a planned activity or intervention engaging PNs or CHWs or involving workforce development.

Beginning with a summative approach to content analysis $^{39}$ and later using an inductive approach we (E.A.R. and R.M.) open-coded text, according to specific areas of $\mathrm{PN}$ and/or CHW intervention (e.g., screening, survivorship, and cancer type). We (E.A.R., R.M., and J.T.) developed a detailed codebook that defined codes and specified how to apply them to text. One researcher (R.M.) had the primary responsibility of coding content according to codebook rules, using NVivo10 ${ }^{\circledR}$ (Victoria, Australia) to manage the data, but had frequent meetings with other investigators (E.A.R. and J.T.) to review analysis. Analysis first assessed whether action plans included $\mathrm{PN}$ and/or $\mathrm{CHW}$ language. For those action plans that did contain $\mathrm{PN}$ and/or CHW language, we coded to reflect the context in which the PN and/or CHW language was used. We used descriptive statistics to characterize overall results and calculated chi-squares to determine differences in $\mathrm{PN}$ and/or $\mathrm{CHW}$ activities and settings among programs.

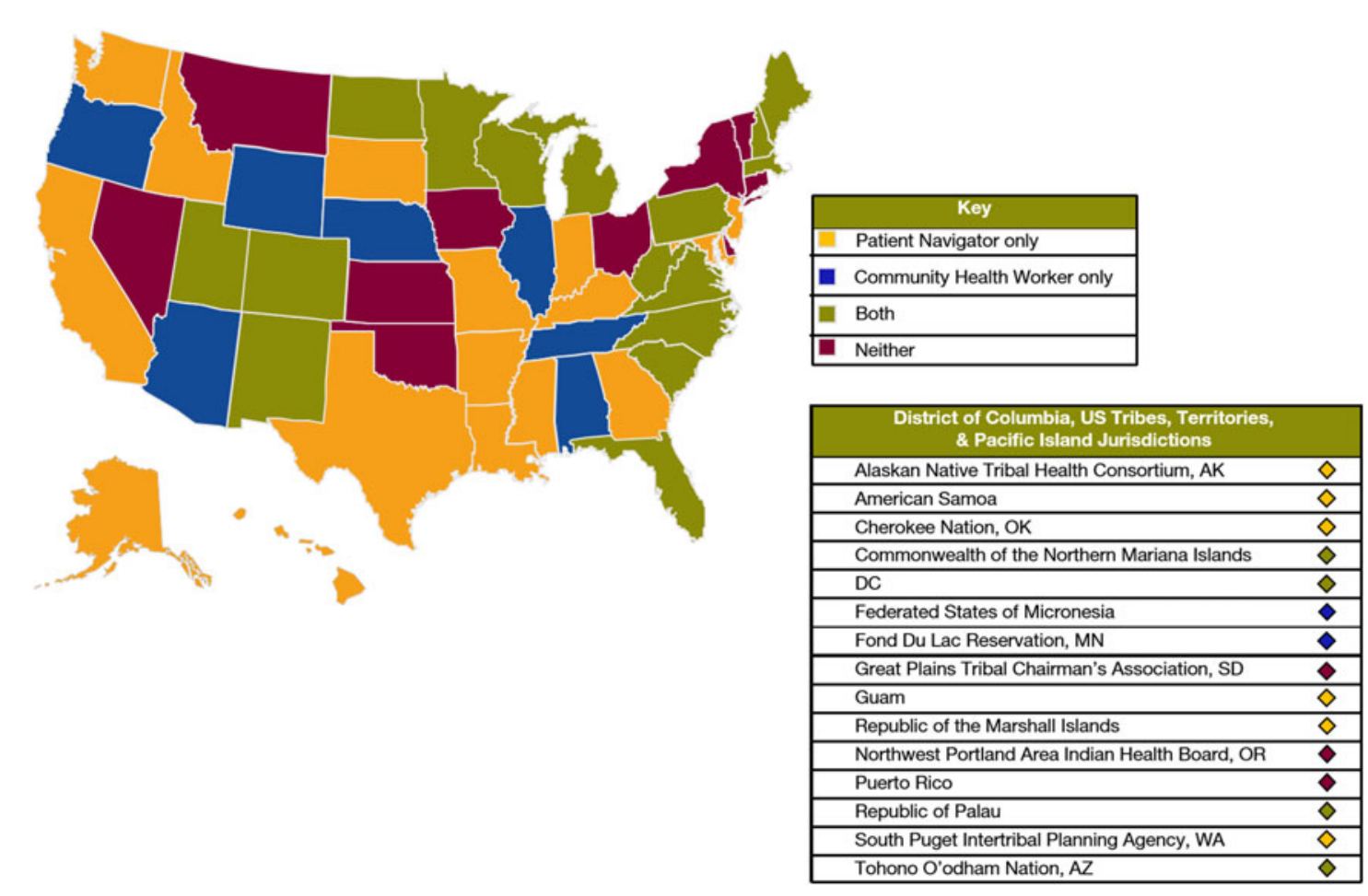

FIG. 1. U.S. map depicting states with action plans using patient navigator and/or community health worker terms. 
Table 1. Percentage of Action Plans with Patient Navigation and/or Community Health Worker Language by Type of Cancer $(n=53)$

\begin{tabular}{lc}
\hline Type of cancer or chronic disease & $\boldsymbol{n}(\%)$ \\
\hline Cancer not specified & $19(36)$ \\
Breast & $25(47)$ \\
Cervical & $24(45)$ \\
Colorectal & $21(40)$ \\
Lung & $3(6)$ \\
Prostate & $3(6)$ \\
Chronic disease (including cancer) & $17(32)$ \\
\hline
\end{tabular}

Note: Percentages do not total $100 \%$ because action plans may have specified more than one cancer type.

\section{Results}

Figure 1 depicts whether action plans from each NCCCP awardee contained PN content only, CHW content only, both, or neither. Twenty-three (35\%) of plans contained PN language only, 9 (14\%) contained CHW language only, $20(31 \%)$ contained PN and CHW content, and 13 plans (20\%) had no language related to $\mathrm{PN}$ or CHW. A total of $82 \%$ $(n=53)$ of 65 NCCCP action plans had content related to $\mathrm{PN}$ and/or $\mathrm{CHW}$ activities. Of those 53 with $\mathrm{PN}$ and/or CHW language, more action plans contained PN $(83 \% ; n=44)$ than CHW $(58 \% ; n=31)$ language. Among plans that contained either $\mathrm{PN}$ or CHW language, this pattern held true in that 22 mentioned only PN (42\%) and 9 mentioned only $\mathrm{CHW}$ (17\%) language.

\section{Cancer type and cancer continuum}

While 36\% $(n=19)$ of action plans with $\mathrm{PN}$ and/or CHW language did not specify which type(s) of cancers PNs/CHWs would work to prevent or control (25\%; $n=13)$, those that did most commonly cited breast $(47 \% ; n=25)$, cervical $(45 \% ; n=24)$, and colorectal $(40 \% ; n=21)$ cancers (Table 1). Additionally, $32 \%$ of action plans described $\mathrm{PN}$ and/or CHW language in relation to chronic diseases in addition to cancer. Furthermore, action plans specified intervening at various points across the cancer continuum (Fig. 2), but primarily with cancer screening $(60 \% ; n=32)$ and survivorship $(55 \% ; n=29)$. Examples of interventions include promoting cancer screening by providing tailored education and arranging transportation services and ensuring survivors are aware of and have access to survivorship resources and participating in survivorship committees at cancer centers.

\section{PN/CHW patient-level and systems-level activities}

Within action plans with $\mathrm{PN}$ and/or $\mathrm{CHW}$ language, PNs/CHWs were most often involved in these patientlevel activities: addressing barriers $(43 \% ; n=23)$, patient education $(36 \% ; n=19)$, outreach $(36 \% ; n=19)$, resource dissemination $(25 \% ; n=13)$, and advocacy (21\%; $n=11$; Fig. 3). They were relatively less frequently providing psychosocial support $(9 \% ; n=5)$, or discussing clinical trials $(8 \% ; n=4)$. PNs/CHWs also were linked with systems-level activities (Fig. 4),

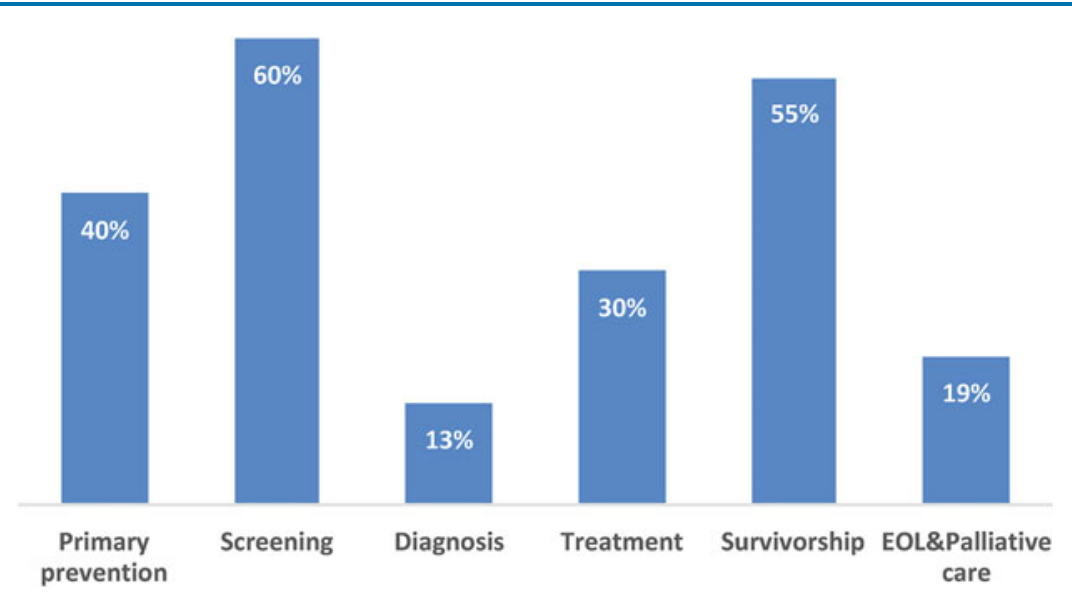

FIG. 2. Percentage of action plans with $P N$ and/or CHW language by cancer continuum area $(n=53)$. Note: Percentages do not total $100 \%$ because action plans may have specified more than one area across the cancer continuum. CHW, community health worker; EOL, end-of-life; PN, patient navigation; PSE, policy, systems, and environmental change. 


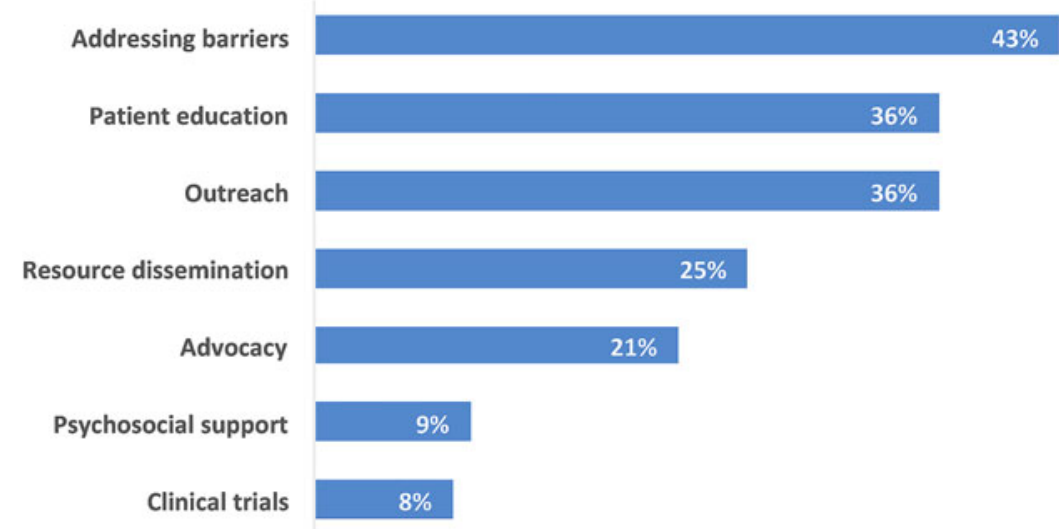

FIG. 3. Percentage of action plans with $\mathrm{PN}$ and/or $\mathrm{CHW}$ language related to individual patient-level activities $(n=53)$. Note: Percentages do not total $100 \%$ because action plans may have specified more than one activity.

such as evaluation $(53 \% ; n=28)$; PSE $(42 \% ; n=22)$; reducing disparities $(40 \% ; n=21)$; and cultural competence $(23 \% ; n=12)$. Table 2 shows examples of PN and/or CHW language from action plans, along with corresponding codes, related to both patient-level and systems-level activities.

\section{Workforce development}

Most $(81 \% ; n=43)$ of the action plans with PN and/or $\mathrm{CHW}$ language reported their programs were engaged in developing the $\mathrm{PN}$ and/or CHW workforce (Table 3$)$. The majority $(88 \% ; n=38)$ of these described engaging in training of PNs and/or CHWs, and over half $(58 \% ; n=25)$ mentioned work related to building
PN and/or CHW capacity within their jurisdiction. Other workforce development activities reported in the action plans related to financing $(35 \% ; n=15)$, networking $(35 \% ; n=15)$, and standardizing either by working toward uniformity in definitions, roles, and core competencies for PNs and/or CHWs (21\%; $n=9)$, or by credentialing $(16 \% ; n=7)$.

\section{$\mathrm{PN} / \mathrm{CHW}$ role differentiation}

Table 4 illustrates that the majority of action plan language showed no statistical differences in role differentiation between PNs or CHWs; however, there are some exceptions. Action plan language more often described engaging CHWs in outreach, patient education,

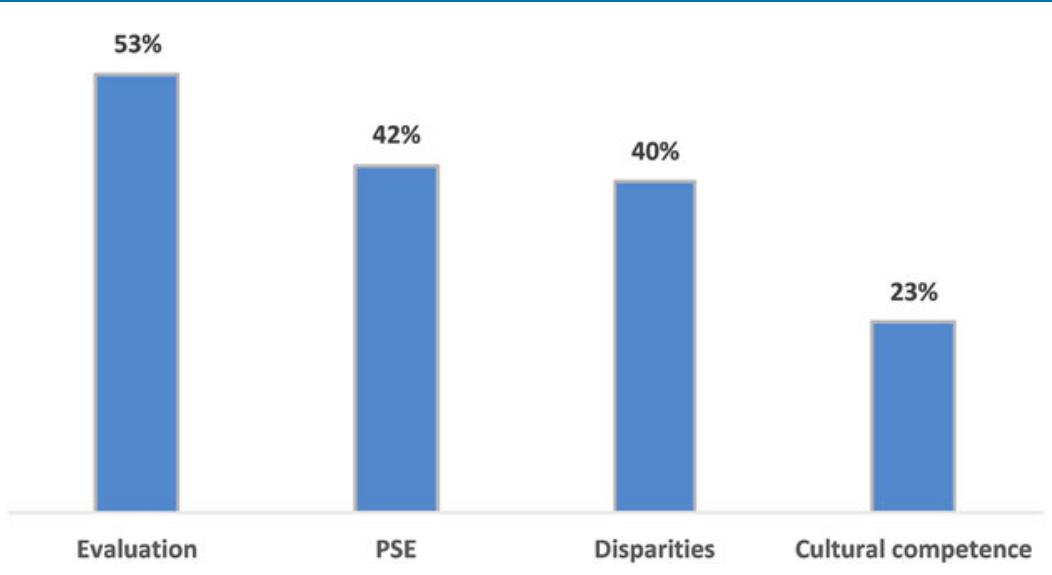

FIG. 4. Percentage of action plans with PNs and/or CHW language related to systems-level activities $(n=53)$. PSE, policy, systems, and environmental change. 
Table 2. Examples of Patient Navigation and/or Community Health Worker Activities in Action Plans

\section{PN/CHW activity \\ or area}

Action plan examples

Addressing barriers [To reduce the need for palliative care patients to travel long distances], the Cancer Program [will] pilot...our long-distance palliative care appointment system. The oncologist will work with community health workers [who will be at the patient's home and] order necessary tests, [talk] with family members about daily care, and respond to the cares and concerns of the patient.

Utilizing the promotora model, staff provides education on the importance of breast and cervical cancer screenings throughout the community and help women in underserved populations access free or low-cost services...Patient navigators helped women access screening programs by helping them complete necessary paperwork, finding transportation to screening appointments (including carpools), and providing translation services when needed. They also provided reminder phone calls to women with screening appointments... and provided culturally sensitive breast health education and navigation services.

Cultural competence

Disparities

PSE

Workforce

This activity is focused on outreach implemented by the Cancer Program Outreach Worker to [name of community] women through Circle Of Life: American Cancer Society's Cancer Education Program developed specifically for American Indians...The Circle of Life program provides education and program promotion materials that are culturally appropriated for the service population...The new program is web-based and has the capacity to make it culturally specific to an individual tribal community through artwork and other graphic images and Tribal Health Services information.

Increase the number of Community Health Workers trained in end-of-life issues. The end-of-life training is intended to increase the skills of community health workers and allied health workers to discuss advance care planning, hospice care, palliative care, and other end-of-life issues with their clients. A culturally sensitive end-of-life training curriculum has been developed, and we are communicating with our community partner about hosting at least one training before [the end of the funding period].

Conducting targeted outreach to increase the rate of mammography screening among groups that experience high mortality rates from breast cancer will ensure screenings are provided to those who are disproportionately impacted by the disease... [We will] conduct "Cancer in Your Community" training for community health workers and allied health workers on cancer early detection...Through lecture and interactive teaching strategies, participants learn about cancer and cancer screening, develop skills to teach others about cancer and screening, and learn about cancer resources in their communities.

[The Health Department] contracted with four local communities already implementing chronic disease prevention through policy and systems change to integrate primary cancer prevention into their current efforts...grantees are in four, lowincome, diverse cities in [our area]....all four grantees incorporated information about the link between obesity and cancer into their training for Community Health Workers (CHW), patient navigators, and Volunteer Health Advisors (VHA). [The Health Department also] funds 3 community-based organizations in high-need areas of the state to provide education, patient navigation and linkages to clinical and social support services with the goal of decreasing colorectal, breast, and cervical cancer screening disparities.

Increase the percent of subject matter expert staff time designated for implementation of PSE efforts in survivorship for tribal health systems improvement...In addition to PSE involving an increased role for village Behavioral Health Aides in caring for cancer survivors, the Cancer Program has also been involved with a statewide effort to increase the presence of patient navigators in facilities and as champions in communities.

Increase the number of funding models proposed to PN/CHW Collaborative...To that end [the health department] cosponsored a Patient Navigation and Community Health Work Sustainability Summit....The intent of this gathering was to engage in critical dialog with key stakeholders, health systems representatives, and policy-makers. Participants evaluated successful [state] and national models and developed recommendations for [the state's] sustainable model for patient navigators and community health workers...Based on analysis, develop and share a funding model proposal with the CHW/ PN Collaborative.

Training:

Increase the number of Community Health Workers (CHWs) trained in standard core competencies to increase use of clinical screenings and link community clinical preventive services. Support development of a statewide system to train CHWs in core competences.

Building capacity:

Increase the number of patient navigation systems...to improve quality of life among cancer survivors... hire and train a parttime HOPE cancer navigator to staff the HOPE Center Resource Room... The HOPE cancer navigator will be available to assist anyone affected by cancer, including family and caregivers.

$\mathrm{CHW}$, community health worker; PN, patient navigation; PSE, policy, systems, and environmental change.

cultural competence, community settings, and primary prevention than PNs. Action plan language more often described engaging PNs, rather than CHWs, in relation to cancer survivorship.

\section{Discussion}

This is the first assessment of $\mathrm{PN} / \mathrm{CHW}$ content in NCCCP action plans. The vast majority of NCCCP awardees reported engaging in PN and/or CHW activities, indicating a prioritization of this intervention in cancer prevention and control consistent with cancer PN literature. ${ }^{40}$ Since PN originated within cancer, ${ }^{20}$ it is not surprising that our review showed that this term was more prevalent than $\mathrm{CHW}$ in NCCCP action plans.

Our findings that NCCCP action plans often described engaging $\mathrm{PNs} / \mathrm{CHWs}$ in screening and in 
Table 3. Patient Navigation/Community Health Worker Workforce Development Categories Within National Comprehensive Cancer Control Program Action Plans $(n=43)$

Workforce development category

Training

Building capacity

Financing

Networking

Standardization/uniformity

Standardization/credentialing

relation to breast, cervical, or colorectal cancer are also supported by the literature. A systematic review found that most PN efficacy studies have been conducted in relation to screening for breast, cervical, or colorectal cancers. ${ }^{40}$ Action plans described PNs and $\mathrm{CHWs}$ most often in relation to addressing barriers and providing patient education, which is also consistent with the literature. ${ }^{8}$ Additional evidence is needed for assessing the efficacy of engaging PNs and CHWs in screening for lung cancer or increasing uptake of $\mathrm{HPV}$ vaccination. Since lung cancer screening is complex $^{41,42}$ and HPV vaccination rates remain low in the United States, ${ }^{43,44}$ there may be a role for PNs and or CHWs in one or both of these arenas as well.

Workforce development is an important aspect in advancing the work of PNs and CHWs. ${ }^{45-48}$ NCCCP

Table 4. Action Plan Language Associated with Community Health Workers Versus

Patient Navigators

\begin{tabular}{|c|c|c|}
\hline & \multicolumn{2}{|c|}{ Role } \\
\hline & $\mathrm{CHW}(n=88), n(\%)$ & $\mathrm{PN}(n=107), \mathrm{n}(\%)$ \\
\hline \multicolumn{3}{|l|}{ Patient-level activities } \\
\hline Resource dissemination & $18(21)$ & $13(12)$ \\
\hline Outreach* & $31(35)$ & $15(14)$ \\
\hline Patient education* & $28(32)$ & 19 (18) \\
\hline Addressing barriers & $26(30)$ & $24(22)$ \\
\hline \multicolumn{3}{|l|}{ Systems-level activities } \\
\hline Cultural competence* & $15(17)$ & $7(7)$ \\
\hline Reducing disparities & $21(24)$ & $16(15)$ \\
\hline PSE & $22(25)$ & $26(24)$ \\
\hline Evaluation & $31(35)$ & $32(30)$ \\
\hline \multicolumn{3}{|l|}{ Workforce development } \\
\hline Building capacity & $23(26)$ & $25(23)$ \\
\hline Financing & $14(16)$ & $17(16)$ \\
\hline Networking & $14(16)$ & $20(19)$ \\
\hline Training & $41(47)$ & $55(51)$ \\
\hline \multicolumn{3}{|l|}{ Setting } \\
\hline Community* & $32(36)$ & $9(8)$ \\
\hline Clinic & $13(15)$ & $27(25)$ \\
\hline \multicolumn{3}{|l|}{ Cancer continuum } \\
\hline Primary prevention* & $28(32)$ & $17(16)$ \\
\hline Screening & $36(41)$ & $33(31)$ \\
\hline Survivorship* & $16(18)$ & $45(42)$ \\
\hline
\end{tabular}

*Indicates significant difference, $p<0.05$. action plans reflected the many ways programs are working to develop and build capacity within PN and $\mathrm{CHW}$ workforces by providing trainings and networking opportunities, working to secure solid sources of financing, working to standardize roles and competencies, and working toward credentialing. Not every NCCCP awardee reported being involved in each of these workforce development activities, but it is clear that many awardees have prioritized taking part in $\mathrm{PN}$ and/or CHW workforce development to increase the capacity of PNs and CHWs to serve populations within their jurisdictions.

Finally, our findings showed that action plans used $\mathrm{CHW}$ language more often related to outreach, patient education, cultural competence, community settings, and primary prevention while action plans used PN language more often related to cancer survivorship. This is consistent with the established perception that $\mathrm{CHWs}$ are trusted members of the communities they serve. ${ }^{29,45,46,49} \mathrm{PN}$ was pioneered in the field of oncology ${ }^{20}$; the association of PNs with individuals postdiagnosis (cancer survivors) seems consistent with this history.

\section{Limitations}

While providing detailed information about $\mathrm{PN}$ and/or CHW activities within NCCCP, action plans do not reflect all that is being done in cancer by PNs and/or CHWs. Specific information may not be contained in NCCCP action plans, either for timing reasons (only 3 years of action plans were examined during the 5year funding cycle) or reporting reasons (an NCCCP awardee may not report all $\mathrm{PN} / \mathrm{CHW}$-related activities they are engaged in, especially if financial resources for the activities are shared across health department entities or programs). ${ }^{45}$

Even though action plans are semi-structured, each one varies in terms of the depth and relevancy of information provided. Therefore, we may not have captured all activities or have enough detail to adequately capture the focus of the activity. Although unlikely, since most NCCCPs had PNs and/or CHW content, we could have missed retrieving content that used an alternative abbreviation for PN or CHWs. These data are also self-reported and potentially could be subject to social desirability bias. Finally, NCCCP awardees did not always specify in their action plans which cancer populations their $\mathrm{PN}$ and/or CHW efforts were focused on; this created difficulty in assessing the specific nature of this work in increasing health equity in underserved populations. 


\section{Strengths}

As noted, this is the first study to quantify PN and CHW activity in cancer across the NCCCP and can be used as a baseline in future analyses. Additionally, the coding of action plan content followed a rigorous process that involved all 3 researchers to minimize bias to the greatest extent possible in a content analysis.

\section{Conclusion}

Understanding how NCCCP awardees engage PNs and CHWs, including awardees' needs for workforce development in this area, can help CDC provide more focused technical assistance to NCCCP awardees as they work to support cancer prevention and screening, improve the quality of life among cancer survivors, and help reduce disparities in cancer prevention and control. This review can serve as a baseline assessment of $\mathrm{PN} / \mathrm{CHW}$ activities in CCC plans and give some indication as to the integration of these approaches into comprehensive cancer control efforts. Future $\mathrm{PN} / \mathrm{CHW}$ efforts among NCCCP awardees can include the specific population addressed, which may allow for easier assessment of the effects of PNs/CHWs in reducing health disparities. Reporting on the specific population served may additionally provide best practices for other NCCCP awardees working within similar populations. Overall, our findings highlight similarities and distinguishing characteristics among how the NCCCP awardees engage PNs and CHWs and can help inform the implementation and evaluation of cancer prevention and control efforts within both public health and oncology health care programs that engage PNs and CHWs. Ongoing support of the implementation of $\mathrm{PN}$ and $\mathrm{CHW}$ interventions and continued assessment of their added value in cancer control offers public health programs, including the NCCCP, a means to further improve chronic disease health equity. ${ }^{9}$

\section{Disclaimer}

The findings and conclusions in this article are those of the authors and do not necessarily represent the official position of the Centers for Disease Control and Prevention.

\section{Author Disclosure Statement}

The authors have no financial disclosures to report.

\section{References}

1. DeLancey JO, Thun MJ, Jemal A, et al. Recent trends in Black-White disparities in cancer mortality. Cancer Epidemiol Biomarkers Prev. 2008;17: 2908-2912.
2. Lerro CC, Robbins AS, Fedewa SA, et al. Disparities in stage at diagnosis among adults with testicular germ cell tumors in the National Cancer Data Base. Urol Oncol. 2014;32:23.e15-23.e21.

3. Robbins AS, Chen AY, Stewart AK, et al. Insurance status and survival disparities among nonelderly rectal cancer patients in the National Cancer Data Base. Cancer. 2010;116:4178-4186.

4. Hao $Y$, Landrine $H$, Jemal $A$, et al. Race, neighbourhood characteristics and disparities in chemotherapy for colorectal cancer. J Epidemiol Community Health. 2011;65:211-217.

5. Lengerich EJ, Tucker TC, Powell RK, et al. Cancer incidence in Kentucky, Pennsylvania, and West Virginia: disparities in Appalachia. J Rural Health. 2005;21:39-47.

6. Sineshaw HM, Freedman RA, Ward EM, et al. Black/White disparities in receipt of treatment and survival among men with early-stage breast cancer. J Clin Oncol. 2015;33:2337-2344.

7. Katz ML, Young GS, Reiter PL, et al. Barriers reported among patients with breast and cervical abnormalities in the patient navigation research program: impact on timely care. Womens Health Issues. 2014;24:e155e162.

8. Rohan EA, Slotman B, DeGroff A, et al. Refining the patient navigation role in a colorectal cancer screening program: results from an intervention study. J Natl Compr Canc Netw. 2016;14:1371-1378.

9. Roland KB, Milliken EL, Rohan EA, et al. Use of community health workers and patient navigators to improve cancer outcomes among patients served by federally qualified health centers: a systematic literature review. Health Equity. 2017;1:61-76.

10. Frieden TR. A framework for public health action: the health impact pyramid. Am J Public Health. 2010;100:590-595.

11. Bauer UE, Briss PA, Goodman RA, et al. Prevention of chronic disease in the 21st century: elimination of the leading preventable causes of premature death and disability in the USA. Lancet. 2014;384:45-52.

12. Ko NY, Darnell JS, Calhoun E, et al. Can patient navigation improve receipt of recommended breast cancer care? Evidence from the National Patient Navigation Research Program. J Clin Oncol. 2014;32:2758-2764.

13. DeGroff A, Schroy PC, 3rd, Morrissey KG, et al. Patient navigation for colonoscopy completion: results of an RCT. Am J Prev Med. 2017;53:363372.

14. Wells KJ, Meade CD, Calcano E, et al. Innovative approaches to reducing cancer health disparities: the Moffitt Cancer Center Patient Navigator Research Program. J Cancer Educ. 2011;26:649-657.

15. Kangovi S, Grande D, Trinh-Shevrin C. From rhetoric to reality-community health workers in post-reform U.S. health care. N Engl J Med. 2015; 372:2277-2279.

16. Wells KJ, Rivera MI, Proctor SS, et al. Creating a patient navigation model to address cervical cancer disparities in a rural Hispanic farm worker community. J Health Care Poor Underserved. 2012;23:1712-1718.

17. Wells KJ, Luque JS, Miladinovic B, et al. Do community health worker interventions improve rates of screening mammography in the United States? A systematic review. Cancer Epidemiol Biomarkers Prev. 2011;20: 1580-1598.

18. Gunn C, Battaglia TA, Parker VA, et al. What makes patient navigation most effective: defining useful tasks and networks. J Health Care Poor Underserved. 2017;28:663-676.

19. Oncology Nursing Society, the Association of Oncology Social Work, and the National Association of Social Workers Joint Position on the Role of Oncology Nursing and Oncology Social Work in Patient Navigation (adopted March 2010). Available at http://aosw.org/cms/wp-content/ themes/inovado/pdfs/aosw-public-docs/PR-PositionPatientNav.pdf Accessed October 4, 2017.

20. Freeman HP, Rodriguez RL. History and principles of patient navigation. Cancer. 2011;117(15 Suppl.):3539-3542.

21. Freund KM, Battaglia TA, Calhoun E, et al. Impact of patient navigation on timely cancer care: the Patient Navigation Research Program. J Natl Cancer Inst. 2014;106:dju115.

22. Rodday AM, Parsons SK, Snyder F, et al. Impact of patient navigation in eliminating economic disparities in cancer care. Cancer. 2015;121:40254034.

23. Wells KJ, Battaglia TA, Dudley DJ, et al. Patient navigation: state of the art or is it science? Cancer. 2008;113:1999-2010.

24. Willis A, Reed E, Pratt-Chapman M, et al. Development of a framework for patient navigation: delineating roles across navigator types. J Oncol Navig Surviv. 2013;4:[online] 
25. Rosenthal EL, Brownstein JN, Rush $\mathrm{CH}$, et al. Community health workers: part of the solution. Health Aff (Millwood). 2010;29:1338-1342.

26. American Public Health Association. Support for Community Health Workers to Increase Health Access and to Reduce Health Inequities. Policy Statement 20091. Washington, DC: American Public Health Association, 2009.

27. Brownstein JN, Hirsch GR, Rosenthal EL, et al. Community health workers "101" for primary care providers and other stakeholders in health care systems. J Ambul Care Manage. 2011;34:210-220.

28. Perez LM, Martinez J. Community health workers: social justice and policy advocates for community health and well-being. Am J Public Health. 2008;98:11-14.

29. Health Resources and Services Administration. Community Health Worker National Workforce Study. 2007. Available at https:// bhw.hrsa.gov/sites/default/files/bhw/nchwa/projections/ communityhealthworkforce.pdf Accessed February 25, 2018.

30. Fernandez ME, Gonzales A, Tortolero-Luna G, et al. Effectiveness of Cultivando la Salud: a breast and cervical cancer screening promotion program for low-income Hispanic women. Am J Public Health. 2009;99: 936-943.

31. Paskett E, Tatum C, Rushing J, et al. Randomized trial of an intervention to improve mammography utilization among a triracial rural population of women. J Natl Cancer Inst. 2006;98:1226-1237.

32. Warren-Mears V, Dankovchik J, Patil M, et al. Impact of patient navigation on cancer diagnostic resolution among Northwest Tribal communities. Cancer Educ. 2013;28:109-118.

33. Ollove M. Under Affordable Care Act, Growing Use of "Community Health Workers." Available at www.pewtrusts.org/en/research-and-analysis/ blogs/stateline/2016/07/08/under-affordable-care-act-growing-use-ofcommunity-health-workers Accessed July 18, 2018.

34. Katzen A, Morgan M. Affordable Care Act Opportunities for Community Health Workers. 2014. Available at www.chlpi.org/wp-content/uploads/ 2013/12/ACA-Opportunities-for-CHWsFINAL-8-12.pdf Accessed July 18, 2018.

35. Office of the Surgeon General. National Prevention Strategy. 2011 Available at www.surgeongeneral.gov/priorities/prevention/strategy/ report.pdf Accessed April 3, 2017.

36. Division of Cancer Prevention and Control Centers for Disease Contro and Prevention. National Comprehensive Cancer Control Program (NCCCP). 2017. Available at www.cdc.gov/cancer/ncccp Accessed April 3 2017.

37. Stewart SL, Lakhani N, Brown PM, et al. Gynecologic cancer prevention and control in the National Comprehensive Cancer Control Program: progress, current activities, and future directions. J Womens Health (Larchmt). 2013;22:651-657.

38. Rochester PW, Townsend JS, Given L, et al. Comprehensive cancer control: progress and accomplishments. Cancer Causes Control. 2010;21: 1967-1977.

39. Hsieh HF, Shannon SE. Three approaches to qualitative content analysis. Qual Health Res. 2005;15:1277-1288.

40. Paskett ED, Harrop JP, Wells KJ. Patient navigation: an update on the state of the science. CA Cancer J Clin. 2011;61:237-249.
41. Lewis JA, Petty WJ, Tooze JA, et al. Low-dose CT lung cancer screening practices and attitudes among primary care providers at an Academic Medical Center. Cancer Epidemiol Biomarkers Prev. 2015;24:664-670.

42. Tammemagi MC, Katki HA, Hocking WG, et al. Selection criteria for lungcancer screening. N Engl J Med. 2013;368:728-736.

43. Curtis $C R$, Dorell $C$, Yankey D, et al. National human papillomavirus vaccination coverage among adolescents aged 13-17 years-National Immunization Survey -teen, United States, 2011. MMWR Suppl. 2014;63: 61-70.

44. Stokley S, Jeyarajah J, Yankey D, et al. Human papillomavirus vaccination coverage among adolescents, 2007-2013, and postlicensure vaccine safety monitoring, 2006-2014-United States. MMWR Morb Mortal Wkly Rep. 2014;63:620-624.

45. Centers for Disease Control and Prevention. Addressing Chronic Disease Through Community Health Workers: A Policy and Systems-Level Approach. Available at www.cdc.gov/dhdsp/docs/CHW_brief.pdf Accessed February 25, 2018.

46. Association of State and Territorial Health Officials. Improving Cancer Prevention and Control: How State Health Agencies Can Support Patient Navigators and Community Health Workers. 2012. Available at www.astho.org/Programs/Prevention/Chronic-Disease/Cancer/Materials/ Improving-Cancer-Prevention-and-Control Accessed July 18, 2018.

47. American Public Health Association. Support for Community Health Worker Leadership in Determining Workforce Standards for Training and Credentialing. Policy Statement Number: 21414. 2014. Available at https://apha.org/policies-and-advocacy/public-health-policy-statements/ policy-database/2015/01/28/14/15/support-for-community-healthworker-leadership Accessed February 25, 2018.

48. Allen CG, Brownstein JN, Cole M, et al. Building a framework for community health worker skills proficiency assessment to support ongoing professional development. J Ambul Care Manage. 2018;41:298-307.

49. Friedman AR, Butterfoss FD, Krieger JW, et al. Allies community health workers: bridging the gap. Health Promot Pract. 2006;7(2 Suppl.):96S-107S.

Cite this article as: Rohan EA, McDougall R, Townsend JS (2018) An exploration of patient navigation and community health worker activities across national comprehensive cancer control programs, Health Equity 2:1, 366-374, DOI: 10.1089/heq.2018.0053.

\section{Abbreviations Used}

$\mathrm{CDC}=$ Centers for Disease Control and Prevention

CDMIS $=$ Chronic Disease Management Information System

$\mathrm{CHRs}=$ community health representatives

$\mathrm{CHW}=$ community health worker

$\mathrm{NCCCP}=$ National Comprehensive Cancer Control Program

$\mathrm{PN}=$ patient navigation

PSE $=$ policy, systems, and environmental change

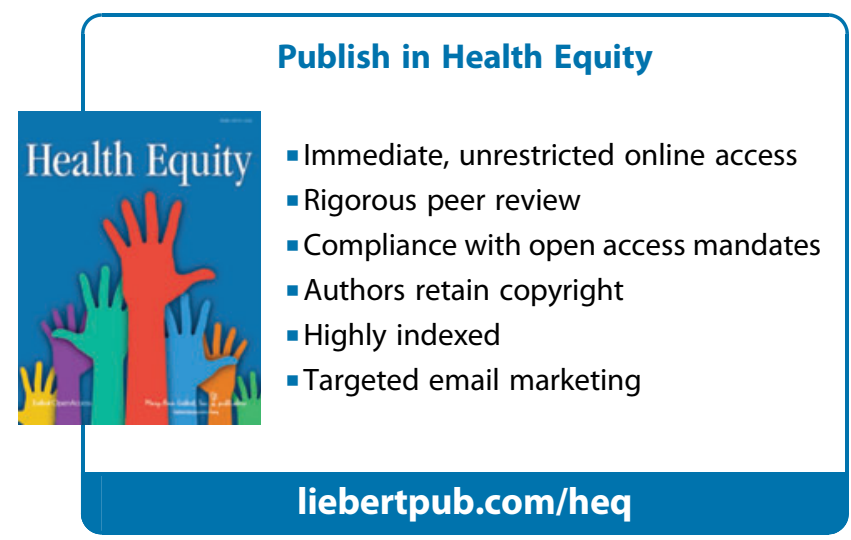

